DESY 09-001

\title{
Prospects for the direct detection of the cosmic neutrino background *
}

\author{
Andreas Ringwald \\ Deutsches Elektronen-Synchrotron DESY, Notkestrasse 85, D-22607 Hamburg, Germany
}

\begin{abstract}
The existence of a cosmic neutrino background - the analogue of the cosmic microwave background - is a fundamental prediction of standard big bang cosmology. Up to now, the observational evidence for its existence is rather indirect and rests entirely on cosmological observations of, e.g., the light elemental abundances, the anisotropies in the cosmic microwave background, and the large scale distribution of matter. Here, we review more direct, weak interaction based detection techniques for the cosmic neutrino background in the present epoch and in our local neighbourhood. We show that, with current technology, all proposals are still off by some orders of magnitude in sensitivity to lead to a guaranteed detection of the relic neutrinos. The most promising laboratory search, based on neutrino capture on beta decaying nuclei, may be done in future experiments designed to measure the neutrino mass through decay kinematics.
\end{abstract}

\section{Introduction}

Over the past decade, we have witnessed extraordinary progress in observational cosmology. In fact, from the interpretation of cosmological data a quite precise knowledge of the energy (mass) budget of the universe emerged. Along with the heavily exploited cosmic microwave background (CMB), standard big bang theory predicts the existence of a cosmic neutrino background (CNB), comprised of relic neutrinos from the epoch of decoupling of weak interactions about one second after the big bang. Presently, the

\footnotetext{
‡ Invited talk presented at PANIC 2008, 9-14 November 2008, Eilat, Israel
} 
inference about or evidence for the existence of the CNB rests solely on cosmological measurements, in particular the light elemental abundances and their interpretation in terms of big bang nucleosynthesis (BBN), the spectrum of $\mathrm{CMB}$ anisotropies, and the large scale matter power spectrum (for a review, see Ref. [1]). All these measurements, however, probe the presence of relic neutrinos only at early stages in the cosmological evolution and, moreover, in a rather indirect way. Here, we will concentrate on more direct, weak interaction based detection possibilities of the CNB, sensitive in particular to the CNB in our local neighborhood and in the present epoch.

\section{Phase space distribution of relic neutrinos}

The design of a direct, weak interaction based detection experiment needs a precise knowledge of the phase space distribution of the relic neutrinos. Its average over large scales has been determined when the primordial plasma had a temperature of about one $\mathrm{MeV}$ : it is given by the homogeneous and isotropic relativistic Fermi-Dirac distribution, $f_{0}=1 /\left(1+\exp \left(p / T_{\nu, 0}\right)\right)$, where $p$ is the modulus of the comoving three-momentum $\mathbf{p}$ and $T_{\nu, 0}=(4 / 11)^{1 / 3} T_{\gamma, 0}=1.95 \mathrm{~K}$ is today's CNB temperature, in terms of the CMB temperature $T_{\gamma, 0}=2.73 \mathrm{~K}$. Correspondingly, the large scale properties of the CNB are tightly related to the properties of the well-measured CMB and are therefore to be considered as fundamental predictions of standard big bang cosmology. Their present number density,

$$
\underbrace{\bar{n}_{\nu, 0}=\bar{n}_{\bar{\nu}, 0}}_{\mathrm{CNB}}=\frac{3}{22} \underbrace{\bar{n}_{\gamma, 0}}_{\mathrm{CMB}}=56 \mathrm{~cm}^{-3},
$$

when summed over all netrino types $i=1,2,3$, is large and comparable to the one of the CMB. Their present average three-momentum, on the other hand, is very small,

$$
\underbrace{\bar{p}_{\nu, 0}=\bar{p}_{\bar{\nu}, 0}}_{\mathrm{CNB}}=3(4 / 11)^{1 / 3} \underbrace{T_{\gamma, 0}}_{\mathrm{CMB}}=5 \times 10^{-4} \mathrm{eV} .
$$

Correspondingly, at least two of the relic neutrino mass eigenstates are non-relativistic today $\left(m_{\nu_{i}} \gg \bar{p}_{\nu_{i}, 0}\right)$, independently of whether neutrino masses have a normal hierarchical or inverted hierarchical pattern. These neutrinos are subject to gravitational clustering into gravitational potential wells due to existing cold dark matter (CDM) and baryonic structures, causing the local neutrino number density to be enhanced relative to the standard value (11) and the momentum distribution to deviate from the one following from the Fermi-Dirac distribution.

A comprehensive and exhaustive study of gravitational clustering of relic neutrinos [2] has revealed that, within the range of possible neutrino masse 11 , one can expect an overdensity of $1 \div 20$ over the mean values in our position in the Milky Way, i.e. at a distance of about $r_{\oplus}=8 \mathrm{kpc}$ from the galactic center (c.f. Fig. 1 (left)). The present day momentum distribution near the Earth is found to be almost isotropic, with mean radial velocity $\left\langle v_{r}\right\rangle \approx 0$ and second moments that satisfy approximately the relation

\footnotetext{
1 The present cosmological limit on the sum of the neutrino masses is in the $\sum_{i} m_{\nu_{i}}<0.5-0.6 \mathrm{eV}$
} range for a $\Lambda \mathrm{CDM}$ model, but can be relaxed by a large factor if more parameters are included [1. 

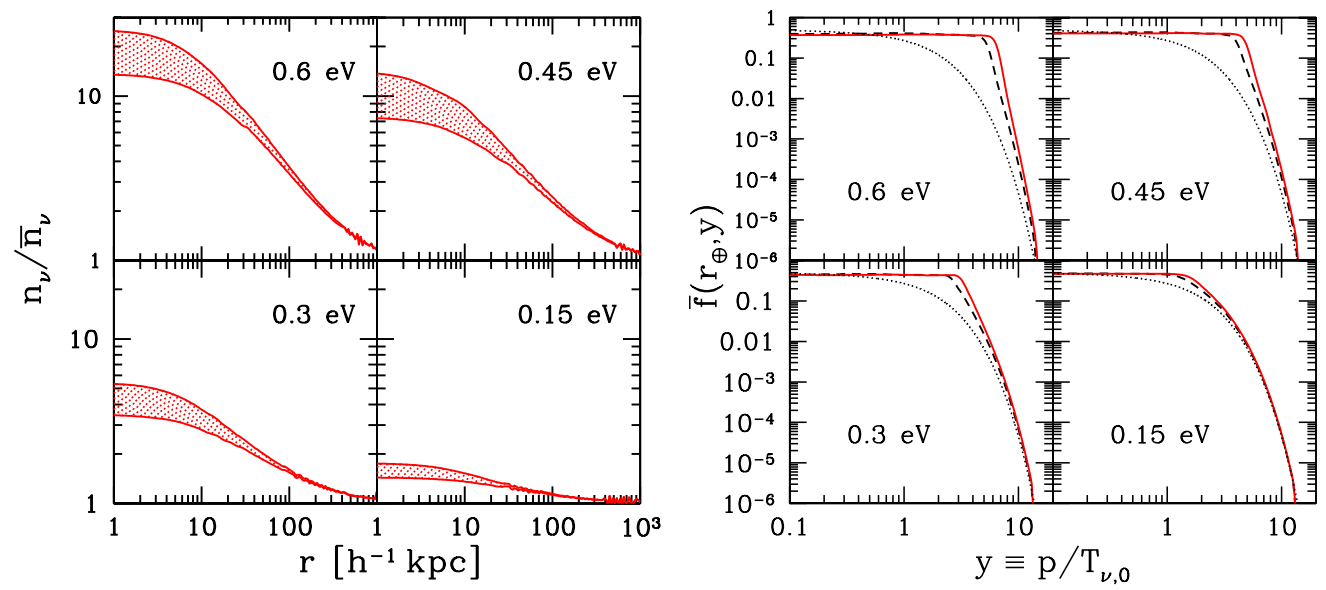

Fig. 1. Characteristics of the CNB in the Milky Way, obtained from solutions of the collisionless Boltzmann equation for the neutrino phase space distribution in the background of the gravitational potential of a NFW CDM halo (NFWhalo) corresponding to the Milky Way and of the present day Milky Way mass distribution (MWnow) [2]. The results are displayed for different values of the neutrino mass, ranging from 0.15 to $0.6 \mathrm{eV}$. Left panels: Neutrino number density profiles, normalized to their cosmological mean, as a function of the distance to the galactic center. The top (bottom) curves correspond to the MWnow (NFWhalo) gravitational potential. Right panels: Momentum distribution of the relic neutrinos in the local neighborhood of the Earth, obtained from the MWnow (solid) and NFWhalo (dashed) potential, approaching for large momenta the relativistic Fermi-Dirac distribution (dotted).

$2\left\langle v_{r}^{2}\right\rangle \approx\left\langle v_{T}^{2}\right\rangle$. The coarse-grained phase space densities $\bar{f}\left(r_{\oplus}, p\right)$ in Fig. 1 (right) are flat at low momenta, with a common value of nearly $1 / 2$, have a turning point at about the escape momenta corresponding to the gravitational potential, and quickly approach the Fermi-Dirac distribution for larger momenta.

\section{Direct CNB detection techniques and their prospects}

In this section we will contentrate on detection techniques of the CNB which are based on weak interaction scattering processes involving the relic neutrinos as beam or as target.

\subsection{Detection via mechanical force due to relic neutrino elastic scattering off target}

The Earth is moving through the almost isotropic (cf. last section) relic neutrino background. Coherent scattering of the corresponding relic neutrino flux off target matter in a terrestrial detector will lead to a mechanical force [34] which may be detected in Cavendish-type torsion balances (cf. Fig. 2) by searching for an annual modulation of the signal. In fact, a terrestrial detector of mass density $\rho_{\mathrm{t}}$ and linear size $r_{\mathrm{t}}<\lambda$, where

$$
\lambda=1 /\langle p\rangle=0.12 \mathrm{~cm} /\left\langle p / T_{\nu, 0}\right\rangle,
$$

is the de Broglie wavelength of the relic neutrinos, will experience a neutrino wind induced acceleration due to coherent scattering [34151617, 


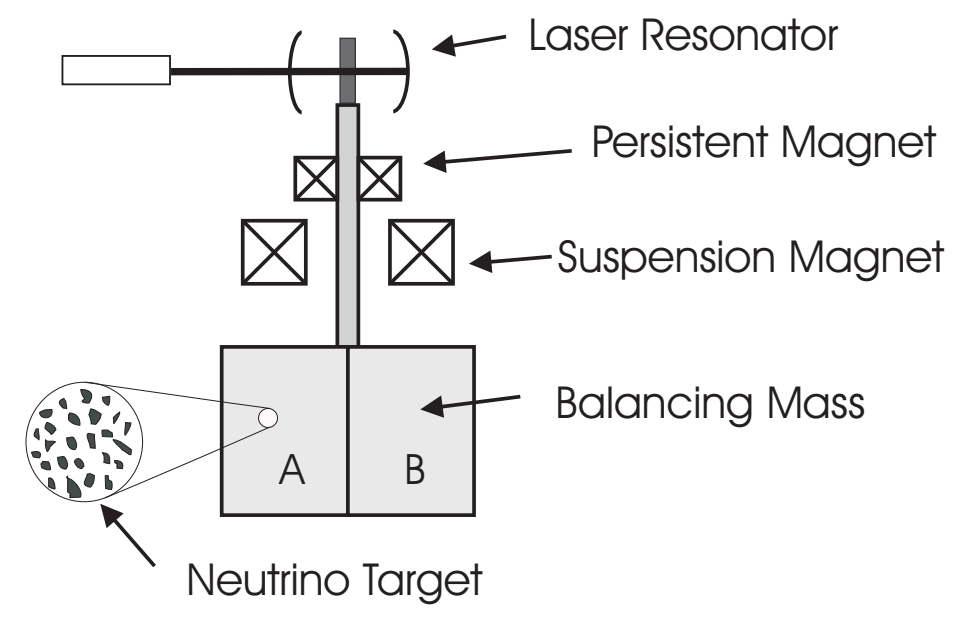

Fig. 2. Cavendish-type torsion balance for relic neutrino detection [6].

$$
\begin{aligned}
a_{\mathrm{t}} & \simeq \sum_{\nu, \bar{\nu}} \underbrace{n_{\nu} v_{\text {rel }}}_{\text {flux }} \frac{4 \pi}{3} N_{A}^{2} \rho_{\mathrm{t}} r_{\mathrm{t}}^{3} \sigma_{\nu N} \underbrace{2 m_{\nu} v_{\text {rel }}}_{\text {mom. transfer }} \\
& \simeq 2 \times 10^{-28} \frac{\mathrm{cm}}{\mathrm{s}^{2}}\left(\frac{n_{\nu}}{\bar{n}_{\nu}}\right)\left(\frac{10^{-3} c}{v_{\text {rel }}}\right)\left(\frac{\rho_{\mathrm{t}}}{\mathrm{g} / \mathrm{cm}^{3}}\right)\left(\frac{r_{\mathrm{t}}}{\lambda}\right)^{3},
\end{aligned}
$$

where $N_{A}$ is Avogadro's number, $\sigma_{\nu N} \simeq G_{F}^{2} m_{\nu}^{2} / \pi$ is the elastic neutrino nucleon cross section, and $v_{\text {rel }}=\left\langle\left|\mathbf{v}-\mathbf{v}_{\oplus}\right|\right\rangle$ is the mean velocity of the relic neutrinos in the rest system of the detector. Here $v_{\oplus} \simeq 7.7 \times 10^{-4} c$ denotes the velocity of the Earth through the Milky Way. The acceleration (4) can be improved further by using foam-like [3] or laminated [4] materials, or by embedding grains of size $\lambda$ (with spacing $\sim \lambda$ ) randomly in a low density host material [8] (cf. Fig. 2). In this way one may exploit a target size much larger than $\lambda$, while still avoiding destructive interference. For Majorana type neutrinos, the acceleration has a further suppression factor $\left(v_{\mathrm{rel}} / c\right)^{2} \simeq 10^{-6}$ for an unpolarized and $v_{\text {rel }} / c \simeq 10^{-3}$ for a polarized target $[6$, respectively.

To infer the prospects of this CNB detection method we note that presently Cavendishtype torsion balances routinely reach an acceleration sensitivity of $10^{-13} \mathrm{~cm} / \mathrm{s}^{2} 9$. Improvements obtainable with current technology may improve this down to $10^{-23} \mathrm{~cm} / \mathrm{s}^{2} 6$ (cf. Fig. 2). Correspondingly, at present this method falls short in sensitivity by at least three orders of magnitude. But it can still be envisaged in the not-so-distant future, say, within thirty to forty years. For the (most likely) case that the neutrinos are of Majorana type, however, the opportunities of CNB detection via this method seem to be very slim.

\subsection{Detection via relic neutrino capture on radioactive nuclei}

A very promising technique for direct detection of the CNB may be realized in beta decay experiments initially designed to measure the neutrino mass 2 through the kinematics of the decay 11121314 . It takes advantage of the fact that the rate for relic

$\overline{2}$ For an interesting proposal to detect relic neutrinos via Pauli blocking effects near thresholds for atomic neutrino pair mission enhanced by laser irradiation, see Ref. [10]. 


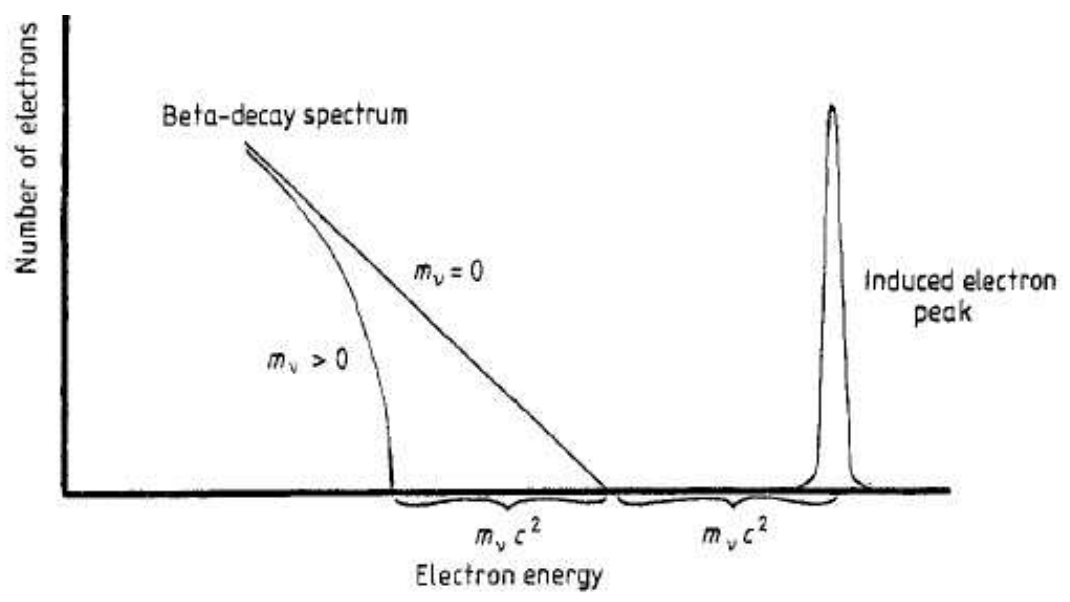

Fig. 3. Idealized electron spectrum for tritium beta decay plus relic neutrino capture from Ref. [11.

neutrino capture on beta decaying nuclei, e.g. on tritium, $\nu_{i}+{ }^{3} \mathrm{H} \rightarrow e+{ }^{3} \mathrm{He}$, converges to a finite value for $v_{\nu} \rightarrow 0$, e.g.

$$
N_{i, \mathrm{CNB}} \simeq 6.5 \mathrm{yr}^{-1}\left(100 \mathrm{~g}^{3} \mathrm{H}\right)^{-1}\left|U_{e i}\right|^{2} \frac{n_{\nu_{i}}}{\bar{n}_{\nu_{i}}},
$$

where $\left|U_{e i}\right|^{2}$ is the electron neutrino content of $\nu_{i}$. Correspondingly, for a sufficient amount of beta decaying target material, the capture rate is reasonably large. Furthermore, because of the non-zero neutrino mass, this process has also a unique signature provided by monoenergetic electrons with kinetic energy $Q_{\beta}+m_{\nu_{i}}$, where $Q_{\beta}$ is the energy release for beta decay with $m_{\nu_{i}}=0$, e.g. $Q_{\beta}=18.6 \mathrm{keV}$ for tritium. This is illustrated in Fig. 3 for an idealized situation where $\left|U_{e i}\right|^{2}=\delta_{e i}$ and where the detector energy resolution as well as experimental background has been neglected.

What are the prospects of this technique? At presently developed beta decay experiments, the relatively small amount of target material leads to a tiny total rate of relic neutrio capture. Furthermore, the energy resolution is still too large, $\Delta E \gtrsim m_{\nu_{i}}$, and the background rate is too high to expect a discovery of the CNB in the very near future. For example, the tritium beta decay experiment KATRIN [15] will exploit in its first phase starting in 2012 effectively $\sim 5 \times 10^{18} \mathrm{~T}_{2}$ molecules, corresponding to $\sim 5 \times 10^{-5} \mathrm{~g}$ tritium [16], is foreseen to have an energy resolution $\Delta E \sim 1 \mathrm{eV}$ and a background rate of about $10 \mathrm{mHz}$. Assuming a neutrino mass $m_{\nu_{i}}=0.6 \mathrm{eV}$ and taking gravitational clustering into account, a $5 \sigma$ evidence for the CNB may be achieved in one year if it were possible, in the second phase of KATRIN, which aims at $\Delta E \sim 0.2 \mathrm{eV}$ and a background rate of $1 \mathrm{mHz}$, to increase the effective mass of tritium to $\sim 35 \mathrm{~g}$ [17]. The ${ }^{187} \mathrm{Re}$ beta decay experiment MARE [18, on the other hand, foresees to have, in the year $\sim 2011$, $10^{5}$ micro-calorimeters of $1-5 \mathrm{mg}$, which is still 4-6 orders of magnitude below the mass needed for a reasonable capture rate [17. But the micro-calorimetric approach seems to be scalable quite easily. 


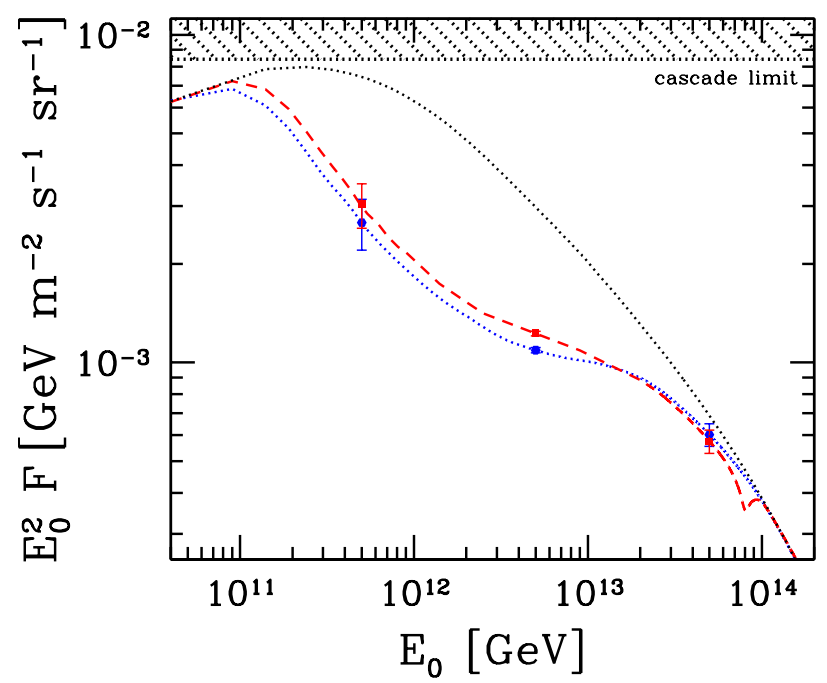

Fig. 4. EHEC $\nu$ spectrum from decaying superheavy $\left(m_{X}=10^{16} \mathrm{GeV}\right)$ particles radiated off cosmic strings (cf. Ref. 23]), taking into account relic neutrino absorption for a hierarchical neutrino spectrum with $m_{\nu_{1}}=10^{-5} \mathrm{eV}, m_{\nu_{2}}=8.3 \times 10^{-3} \mathrm{eV}, m_{\nu_{3}}=5.2 \times 10^{-2} \mathrm{eV}$ (dashed line). The error bars correspond to the combined projected sensitivity of the ANITA 25] and LOFAR 26] EHEC $\nu$ detectors.

\subsection{Detection via absorption features in extremely energetic cosmic neutrino spectra}

An appealing opportunity to catch a glimpse of the CNB in the present epoch emerges from the possible existence of extremely high-energy cosmic neutrinos (EHEC $\nu$ 's), originating e.g. from decaying superheavy particles which are radiated off topological defects generated at phase transitions in the very early universe (cf. Fig. 4). EHEC $\nu$ s can annihilate with relic anti-neutrinos (and vice versa) into $Z$ bosons, $\nu \bar{\nu} \rightarrow Z$, if their energies coincide with the respective resonance energies,

$$
E_{0, i}^{\mathrm{res}}=\frac{m_{Z}^{2}}{2 m_{\nu_{0, i}}}=4.2 \times 10^{12}\left(\frac{\mathrm{eV}}{m_{\nu_{i}}}\right) \mathrm{GeV},
$$

where $m_{Z}$ denotes the $Z$ mass. An exceptional loss of transparency of the CNB for cosmic neutrinos results from the fact that the corresponding annihilation cross-section on resonance is enhanced by several orders of magnitude with respect to non-resonant scattering. As a consequence, the diffuse EHEC $\nu$ flux arriving at Earth is expected to exhibit absorption dips [19|20|21|22]23|24] whose locations in the spectrum are determined by the respective resonance energies of the annihilation processes. Provided that the dips can be resolved on Earth, they could produce the most direct evidence for the existence of the CNB so far. Promisingly, EHEC $\nu$ detectors such as ANITA [25], LOFAR [26] and SKA [27], which will operate within the next decade, have a very good sensitivity in the relevant energy range. In fact, provided that the actual EHEC $\nu$ flux is close to the current bounds, relic neutrino absorption spectroscopy can become a realistic possibility in the near future (cf. Fig. (4). 


\section{References}

[1] S. Hannestad, Ann. Rev. Nucl. Part. Sci. 56 (2006) 137 arXiv:hep-ph/0602058.

[2] A. Ringwald and Y. Y. Y. Wong, JCAP 0412 (2004) 005 arXiv:hep-ph/0408241].

[3] B. F. Shvartsman et al., JETP Lett. 36 (1982) 277 [Pisma Zh. Eksp. Teor. Fiz. 36 (1982) 224].

[4] P. F. Smith and J. D. Lewin, Phys. Lett. B 127 (1983) 185.

[5] I. Ferreras and I. Wasserman, Phys. Rev. D 52 (1995) 5459.

[6] C. Hagmann, arXiv:astro-ph/9902102 arXiv:astro-ph/9905258

[7] G. Duda, G. Gelmini and S. Nussinov, Phys. Rev. D 64 (2001) 122001 arXiv:hep-ph/0107027.

[8] P. F. Smith, Phil. Trans. Roy. Soc. Lond. A 361 (2003) 2591.

[9] E. G. Adelberger et al., Prog. Part. Nucl. Phys. 62 (2009) 102.

[10] T. Takahashi and M. Yoshimura, arXiv:hep-ph/0703019

[11] J. M. Irvine and R. Humphreys, J. Phys. G 9 (1983) 847.

[12] A. G. Cocco, G. Mangano and M. Messina, JCAP 0706 (2007) 015 arXiv:hep-ph/0703075.

[13] R. Lazauskas, P. Vogel and C. Volpe, J. Phys. G 35 (2008) 025001 arXiv:0710.5312 [astro-ph]].

[14] M. Blennow, Phys. Rev. D 77 (2008) 113014 arXiv:0803.3762 [astro-ph]].

[15] A. Osipowicz et al. [KATRIN Collaboration], arXiv:hep-ex/0109033

[16] C. Weinheimer, talk presented at Workshop on New Instruments for Neutrino Relics and Mass, 8 December 2008, CERN, Geneva/CH, http://indico.cern.ch/confAuthorIndex.py?confId=42884

[17] M. Messina, talk presented at Workshop on New Instruments for Neutrino Relics and Mass, 8 December 2008, CERN, Geneva/CH, http://indico.cern.ch/confAuthorIndex.py?confId=42884

[18] E. Andreotti et al., Nucl. Instrum. Meth. A 572 (2007) 208.

[19] T. J. Weiler, Phys. Rev. Lett. 49 (1982) 234; Astrophys. J. 285, 495 (1984).

[20] B. Eberle, A. Ringwald, L. Song and T. J. Weiler, Phys. Rev. D 70 (2004) 023007.

[21] G. Barenboim, O. Mena Requejo and C. Quigg, Phys. Rev. D 71 (2005) 083002.

[22] J. C. D'Olivo, L. Nellen, S. Sahu and V. Van Elewyck, Astropart. Phys. 25 (2006) 47.

[23] A. Ringwald and L. Schrempp, JCAP 0610, 012 (2006) arXiv:astro-ph/0606316].

[24] O. Scholten and A. van Vliet, JCAP 0806 (2008) 015 arXiv:0801.3342 [astro-ph]].

[25] P. Gorham et al. [ANITA collaboration], arXiv:0812.1920 [astro-ph]; arXiv:0812.2715 [astro-ph].

[26] O. Scholten et al., Astropart. Phys. 26 (2006) 219.

[27] C. W. James and R. J. Protheroe, arXiv:0802.3562 [astro-ph]. 